\title{
The Impact of Iterative Reconstruction on Reducing the Radiation Dose for Coronary Calcium Scoring: An Investigation Using Pulsating Calcified Coronary Phantom
}

\author{
So Ode ${ }^{1}$, Yasuyuki Kobayashi ${ }^{2}$, Yukihisa Nozu ${ }^{1}$, Yasuyoshi Ogawa ${ }^{3}$, \\ Akiyuki Kotoku', Yuki Saito ${ }^{2}$, and Yasuo Nakajima ${ }^{1}$
}

(Received for Publication: August 20, 2016)

\begin{abstract}
Aim: The aim of the present study was to evaluate the effects of radiation dose reduction and iterative reconstruction protocols on coronary artery calcium (Agatston) scores using a pulsating cardiac phantom.

Materials and Methods: We used a pulsating cardiac phantom containing three calcified cubes of different sizes (3-mm diameter: small, 4-mm diameter: medium, and 5-mm diameter: large) and four calcified hydroxyapatite cubes of varying density in order to model coronary artery calcification: $5 \%$ (44HU), $15 \%$ (233HU), $25 \%$ (586HU), and 35\% (792HU). Images were acquired with a 320-slice MDCT (Aquilion ONE ViSION Edition, Toshiba, Nasu, Japan), using ECG gated volume scans with tube potential:120kV, 275 ms rotation time, 60, 70, 100,150 and $240 \mathrm{~mA}$ tube current. The Agatston scores at all dose variations in all five reconstruction methods (filtered back projection [FBP] and adaptive iterative dose reconstruction [AIDR3D]: Weak, Mild, Standard, and Strong) for all three phantom sizes were compared to the reference dose protocol reconstructed with FBP.

Results: When the FBP and AIDR 3D: Weak protocol were used, Agatston scores significantly increased in all phantom sizes as doses of radiation decreased $(\mathrm{p}<0.001)$. When the AIDR3D: Mild, Standard, and Strong protocols were used, no significant differences in Agatston scores were observed for any phantom size among different radiation dose protocols. For all radiation dose protocols, Agatston scores significantly decreased as IR levels increased $(\mathrm{p}<0.003$, respectively). When AIDR 3D: Mild was used, no significant differences in Agatston scores were observed between IR and FBP protocols for any phantom size.

Conclusions: The AIDR3D: Mild protocol allowed for significant reductions in radiation dose relative to standard FBP protocols, without influencing Agatston scores. Therefore, evaluation of CAC score using a combination of low-dose radiation and IR may present a reliable alternative to conventional CAC scoring.
\end{abstract}

\section{Key Words}

coronary calcium scoring, agatston score, iterative reconstruction, dose reduction

\section{Introduction}

Coronary artery disease is the leading cause of death in developed countries. ${ }^{1)}$ The presence of calcification in the coronary arteries is a specific marker of coronary artery disease that can be quantified into a coronary artery calcium (CAC) score using estab- lished computed tomography (CT) methods such as the Agatston score. ${ }^{2)}$ Agatston scoring involves a simple, noninvasive test performed without contrast media. Scores are calculated according to the weighted density of the calcified plaque within the coronary arteries and are useful in stratifying the risk of coronary artery disease. ${ }^{3-4)}$

1 Department of Radiology, St. Marianna University School of Medicine, Kawasaki, Japan

2 Department of Advanced Biomedical Imaging Informatics, St. Marianna University School of Medicine, Kawasaki, Japan

3 Department of Radiological Technology, St. Marianna University School of Medicine, Kawasaki, Japan 
However, CAC scoring using CT involves exposure to radiation. According to a previous study by Kim et al. ${ }^{5)}$, the effective radiation dose may reach as high as $10.5 \mathrm{mSv}$ when standard filtered back projection (FBP) reconstructions are used.

Although the radiation dose required in coronary CT angiography has decreased over the past years $\left.{ }^{6}\right)$ due to the adoption of the "as low as reasonably achievable (ALARA)" principle, there is still a need to keep radiation dose to a minimum due to the relatively common nature of cardiac CT scans. Indeed, current guidelines recommend CAC scoring even for asymptomatic adults at low-to-intermediate and intermediate cardiovascular risk. ${ }^{7)}$ However, cardiac CT that has been reconstructed using standard FBP at lower radiation doses tend to exhibit increased image noise.

Recently, iterative reconstruction (IR) algorithms have become available, allowing for reductions in noise and improved image quality using lower doses of radiation relative to those associated with traditional FBP algorithms. ${ }^{8-10)}$ Furthermore, some ex vivo and in vitro studies have reported no significant differences in CAC score between standard-dose FBP and low-dose IR algorithms when the CAC score is relatively high (300-700). ${ }^{11-13)}$ However, the effects of dose reduction and IR on CAC score re- main uncertain when the CAC score is low. Therefore, the present study aimed to evaluate the effects of radiation dose reduction and IR protocols on CAC (Agatston) scores ranging from low to high using a pulsating cardiac phantom.

\section{Materials and Methods}

\section{Cardiac phantom}

We used a cardiac phantom (Fuyo Co., Tokyo, Japan), which allows researchers to simulate a wide variety of clinical scenarios involving the heart, in order to evaluate the effects of radiation dose reduction on CAC scores. The phantom (Figure 1A) consists of a motor comprised of two rings in an acrylic housing: a larger, proximal ring representing the cardiac base and a smaller, distal ring representing the cardiac apex, and has previously been described in detail. ${ }^{14)}$ The two rings of this phantom rotate and converge to simulate cardiac motion. The frequency of this simulated heart motion can be adjusted between 1 and 150 beats per minute (bpm). In the present study, the frequency of this cyclic action was set at $60 \mathrm{bpm}$ because it approximates the reported average in clinical studies.

Three transparent ABS tubes (Fuyo Co., Tokyo, Japan) with inner diameters of $6.0 \mathrm{~mm}$ and $8.0 \mathrm{~mm}$ were used as coronary artery phantoms and attached

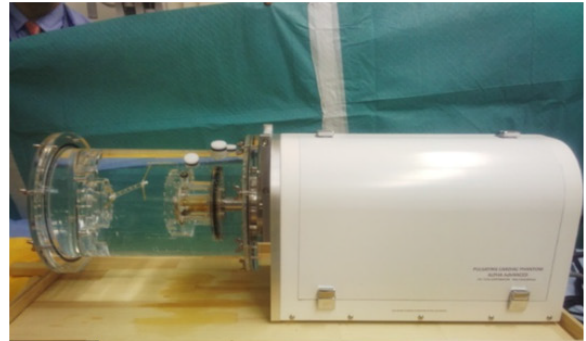

a)

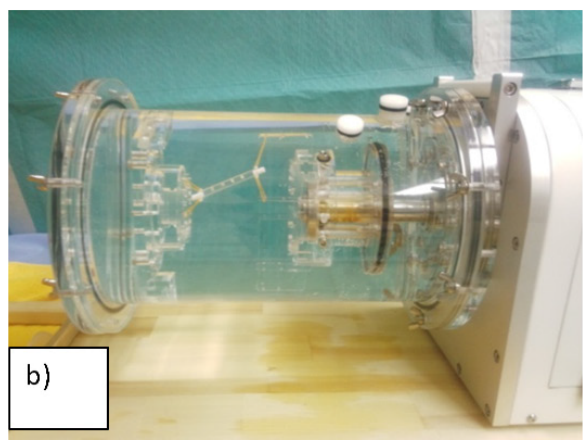

c)
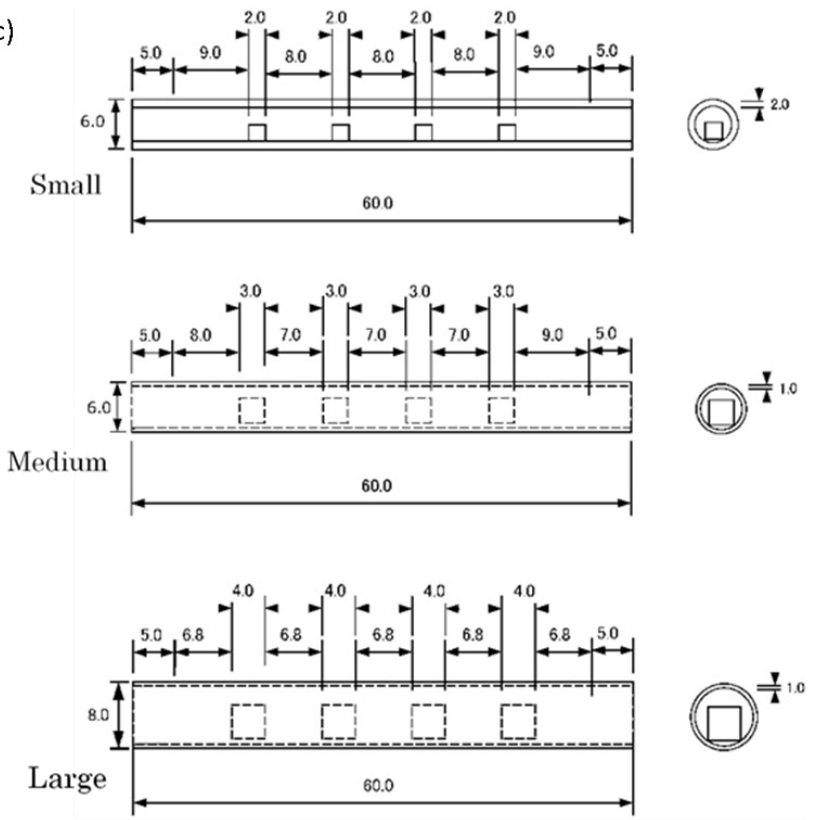

Figure 1. Pulsating cardiac phantom with calcified coronary phantom 
between the two rings of the cardiac phantom. Each tube contained three calcified cubes of different sizes (3-mm diameter: small, 4-mm diameter: medium, and 5-mm diameter: large) and four calcified hydroxyapatite cubes of varying density in order to model coronary artery calcification: $5 \%(44 \mathrm{HU}), 15 \%(233 \mathrm{HU})$, $25 \%$ (586HU), and 35\% (792HU) (Figure 1B). The tubes were filled with diluted glucose solution to ensure a lumen density of approximately 40 Hounsfield units (HU), similar to that of human blood. The precise dimensions and stenosis for each phantom vessel were verified by the manufacturer using precision instruments, with an error of $0.01 \mathrm{~mm}$ or smaller for diameter measurements.

\section{Imaging protocol}

Images were acquired with a 320-slice multi-detector CT (MDCT) scanner (Aquilion ONE ViSION Edition, Toshiba, Nasu, Japan), using ECG-gated volume scans (tube potential: $120 \mathrm{kV}$; rotation time: 275 ms; tube current: $240 \mathrm{~mA}$; routine dose, $150 \mathrm{~mA} ; 38 \%$ reduced dose, $100 \mathrm{~mA} ; 58 \%$ reduced dose, $70 \mathrm{~mA}$; $71 \%$ reduced dose, $60 \mathrm{~mA} ; 75 \%$ reduced dose). The slice thickness was $0.5 \mathrm{~mm}$, and the reconstructed slice thickness was $3.0 \mathrm{~mm}$ according to the standard protocol for the Agatston score. The detector coverage was $16 \mathrm{~cm}$. Scans were repeated 15 times under each condition. The images were reconstructed using filtered back projection (FBP) and four preset algorithms (Weak, Mild, Standard, and Strong) in the Adaptive Iterative Dose Reduction 3-Dimensional (AIDR3D) algorithm tool. Kernels for soft tissue re- construction were applied to all images, which have been validated for use in calcium scanning.

\section{Image analysis}

Reconstructed images were transferred to a $\mathrm{ZIO}^{\circledR}$ workstation (ZIOsoft., Tokyo, Japan) for analysis of the CAC score (Agatston score) of the calcified coronary artery phantoms. Agatston scores at all dose variations in all five reconstruction methods (FBP, IR: Weak, Mild, Standard, and Strong) for all phantom sizes (small, medium, large, and total) were compared to the reference dose protocol reconstructed with FBP.

\section{Statistical analysis}

The distributions of all variables were examined. Median and interquartile ranges were calculated for non-normally distributed continuous variables and compared using Wilcoxon signed-rank tests, the Steel's tests, and Jonckheere-Terpstra test for the trends. Statistical calculations were performed using JMP 9 (SAS Institute Inc.) and EZR (http:// www.jichi.ac.jp/saitama-sct/SaitamaHP.files/statmed. html). A two-tailed $\alpha$ of 0.05 was defined as the level of statistical significance for all analyses.

\section{Results}

The median Agatston score for standard-dose FBP was 646.53 (range: 636.48-665.07). The Agatston scores calculated for each radiation dose and IR reconstruction protocol are presented in Table $\mathbf{1}$ and Figures 2-4.

Table 1. Agatston Score, Compared to CONTROL (FBP at routine dose)

\begin{tabular}{|c|c|c|c|c|c|}
\hline & FBP & AIDR3D.WEAK & AIDR3D.MILD & AIDR3D.STANDARD & AIDR3D.STRONG \\
\hline \multicolumn{6}{|c|}{$240 \mathrm{~mA}$ (routine dose) } \\
\hline TOTAL & $637.09(629.02-644.73)$ & 634.42(629.19-643.27) & $631.08(620.00-638.12)$ & $630.30(618.89-637.00)$ & $619.57(614.16-630.39)$ \\
\hline LARGE & $396.62(390.01-402.46)$ & $396.45(390.27-402.03)$ & $394.99(390.27-396.97)$ & 394.73(389.07-396.28) & 395.33(391.13-398.08) \\
\hline MEDI & 205.69(198.91-211.27) & 205.78(199.51-210.42) & 205.18(195.39-206.90) & 204.49(195.48-206.30) & $195.82(190.41-205.26)$ \\
\hline SMAL & $31.76(30.13-32.45)$ & $31.76(30.05-32.11)$ & $31.51(29.62-32.02)$ & $31.25(29.53-31.85)$ & $29.53(28.85-30.56)$ \\
\hline \multicolumn{6}{|c|}{$150 \mathrm{~mA}$ ( $38 \%$ dose reduction) } \\
\hline TOTAL & $636.23(623.61-640.86)$ & 632.11(623.26-639.32) & $627.21(619.40-638.37)$ & $620.00(614.94-628.93)$ & $617.77(610.47-626.61)$ \\
\hline LARGE & $401.00(392.16-404.86)$ & 400.31(391.81-404.61) & 399.37(391.13-404.18) & 396.02(391.64-399.80) & $398.00(392.67-400.74)$ \\
\hline MEDI & 203.72(194.45-208.53) & 203.63(194.19-208.78) & 199.51(193.33-204.32) & 195.56(191.87-202.09) & 190.07(183.63-195.48) \\
\hline SMAL & $30.82(29.79-32.45)$ & $30.82(29.70-32.02)$ & $29.96(29.36-30.99)$ & $29.19(28.33-29.96)$ & $28.59(27.99-29.88)$ \\
\hline \multicolumn{6}{|c|}{$100 \mathrm{~mA}$ ( $58 \%$ dose reduction) } \\
\hline TOTAL & $651.85(644.30-661.90)$ & 647.56(635.88-655.46) & $633.48(622.06-640.61)$ & $627.30(618.80-633.74)$ & $618.54(612.02-625.58)$ \\
\hline LARGE & $402.46(396.54-408.21)$ & 402.55(396.88-407.95) & $401.34(395.25-402.37)$ & $400.40(394.22-403.15)$ & $398.43(394.65-404.18)$ \\
\hline MEDI & 218.06(208.78-221.23) & 215.74(203.89-218.83) & 202.17(192.04-215.05) & 200.11(188.61-201.75) & $190.50(184.58-198.65)$ \\
\hline SMAL & $33.22(31.59-34.77)$ & $32.54(30.48-34.00)$ & $30.39(29.19-31.68)$ & $29.62(27.73-31.16)$ & 28.67(27.39-29.96) \\
\hline \multicolumn{6}{|c|}{$70 \mathrm{~mA}$ ( $71 \%$ dose reduction) } \\
\hline TOTAL & $665.07(649.28-678.64)$ & $654.08(636.31-660.52)$ & $638.20(619.92-645.93)$ & $632.02(619.40-635.88)$ & $620.00(616.14-625.50)$ \\
\hline LARGE & $403.40(400.14-412.59)$ & 403.66(397.57-405.89) & $401.77(398.00-404.43)$ & 399.80(396.79-404.43) & $401.26(398.00-404.86)$ \\
\hline MEDI & $224.58(214.97-237.03)$ & $216.94(208.87-224.92)$ & 206.21(195.48-210.93) & 198.83(192.30-202.17) & $189.98(185.26-196.16)$ \\
\hline SMAL & $33.40(31.33-34.51)$ & $31.08(30.05-33.40)$ & $29.53(28.42-31.76)$ & $29.36(28.24-31.68)$ & $28.76(27.82-31.16)$ \\
\hline \multicolumn{6}{|c|}{$60 \mathrm{~mA}(75 \%$ dose reduction) } \\
\hline TOTAL & $666.19(653.65-675.98)$ & $645.41(634.94-655.80)$ & $635.71(630.39-643.61)$ & $630.13(622.66-637.77)$ & $621.63(614.16-636.31)$ \\
\hline LARGE & $411.30(404.69-414.82)$ & 405.98(397.91-411.99) & $405.98(398.00-410.36)$ & $405.29(397.14-407.53)$ & $402.80(396.28-409.41)$ \\
\hline MEDI & $215.74(211.02-224.67)$ & 208.36(205.01-212.48) & $201.40(194.79-206.38)$ & 195.48(189.38-202.78) & $192.47(183.46-201.83)$ \\
\hline SMAL & $37.52(35.20-42.58)$ & $34.00(30.56-35.11)$ & $32.45(29.53-32.97)$ & $31.76(29.36-32.45)$ & $29.10(27.99-31.76)$ \\
\hline
\end{tabular}


Agatston scores for various radiation doses when IR levels were held constant (Figure 2)

When the FBP protocol was used, Agatston scores significantly increased in total (overall) and small phantom sizes as doses of radiation decreased $(p<0.001)$. When the AIDR3D: Weak protocol was used, Agatston scores significantly increased in total as radiation doses decreased $(\mathrm{p}<0.001)$, but tended to

1) Total Phantom

a) FBP

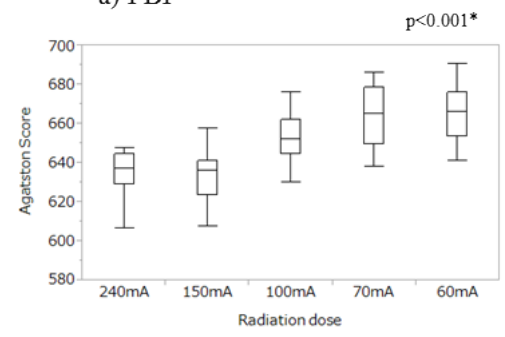

d) STANDARD

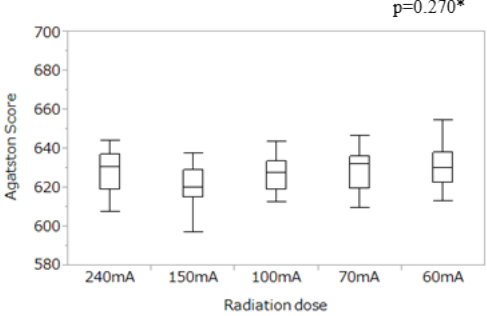

2) Small Phantom

a) FBP

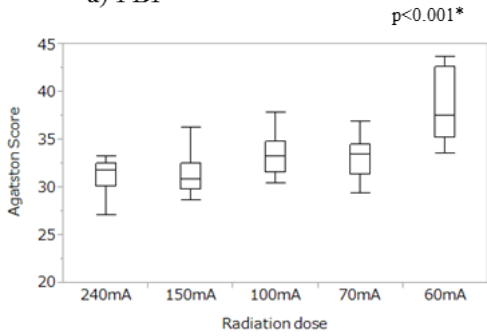

d) STANDARD

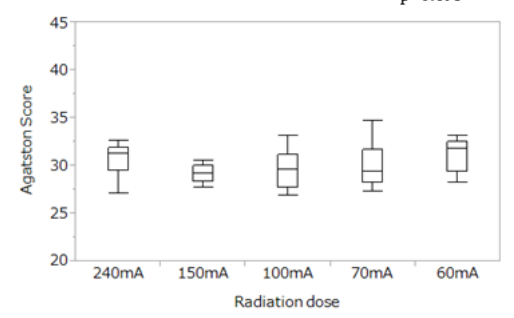

b) WEAK

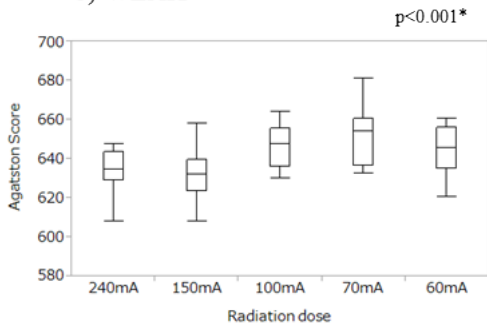

e) STRONG

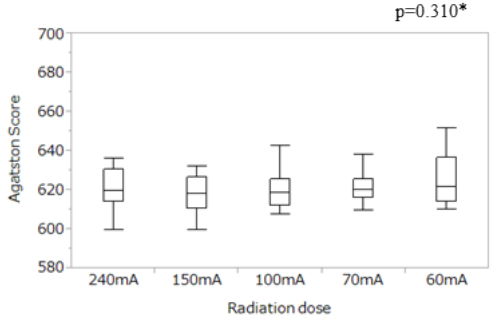

c) MILD

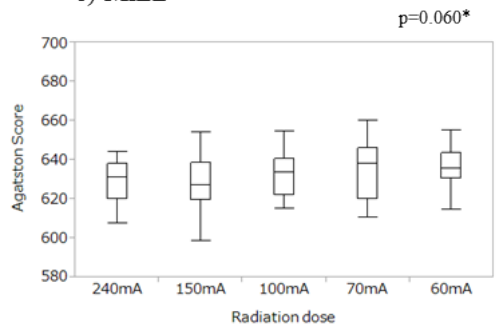

* Jonckheere-Terpstra test b) WEAK

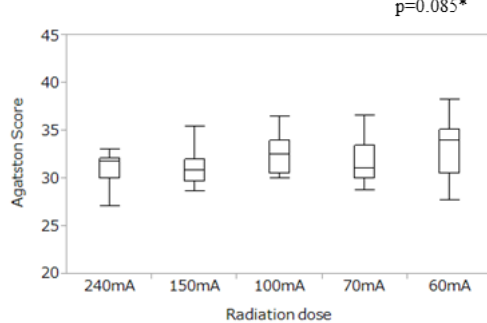

e) STRONG

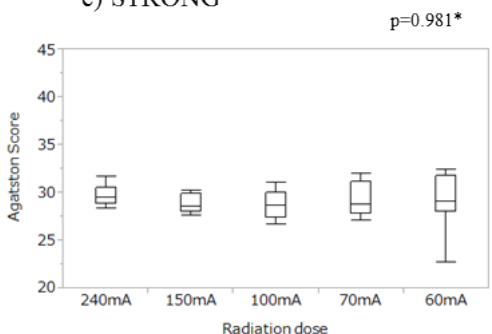

c) MILD

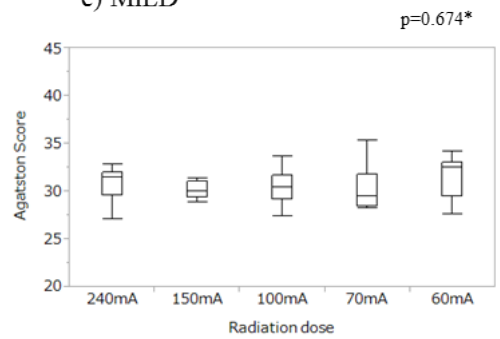

Figure 2. Agatston scores for various radiation doses when IR levels were held constant 
increase in small phantom sizes, though this difference was not significant. When the AIDR3D: Mild, Standard, and Strong protocols were used, no significant differences in Agatston scores were observed for both total and small phantom size among the differ- ent radiation dose protocols.

Agatston scores for various IR levels when radiation dose was held constant (Figure 3)

For all radiation dose protocols, Agatston scores

1) Total Phantom

a) $240 \mathrm{~mA}$ (routine dose)

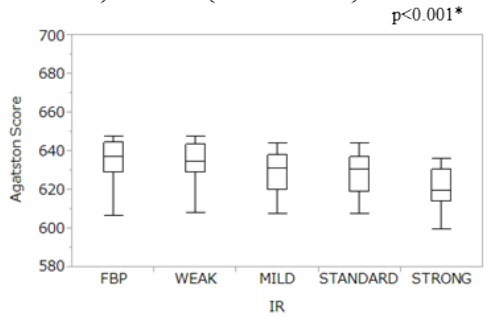

d) $70 \mathrm{~mA}$ ( $71 \%$ dose reduction)

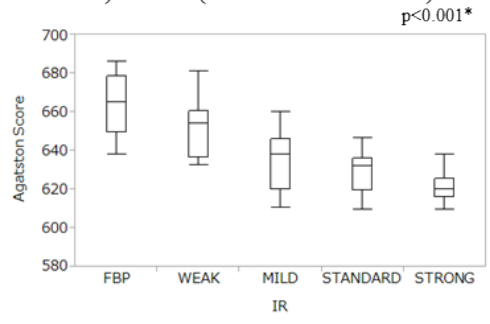

b) $150 \mathrm{~mA}(38 \%$ dose reduction) $\mathrm{p}<0.001^{*}$

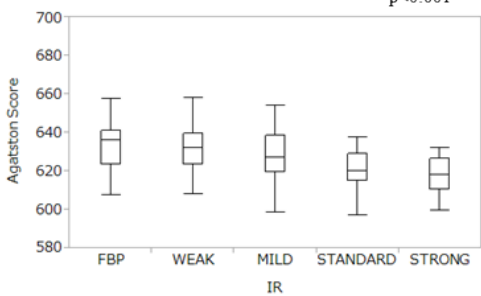

e) $60 \mathrm{~mA}(75 \%$ dose reduction)

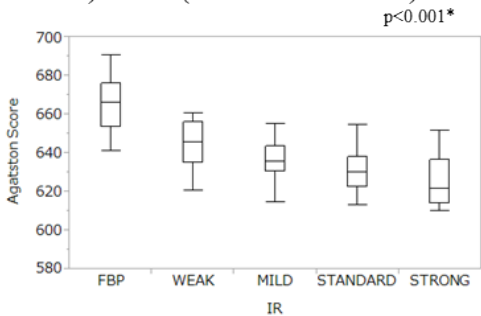

c) $100 \mathrm{~mA}(58 \%$ dose reduction)

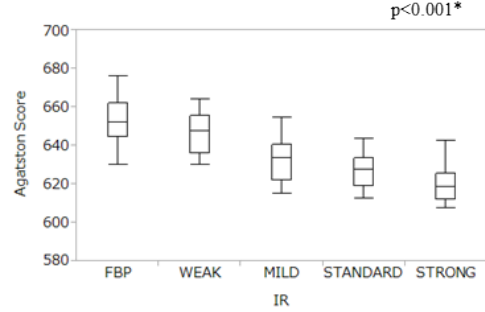

* Jonckheere-Terpstra test

2) Small Phantom

a) $240 \mathrm{~mA}$ (routine dose)

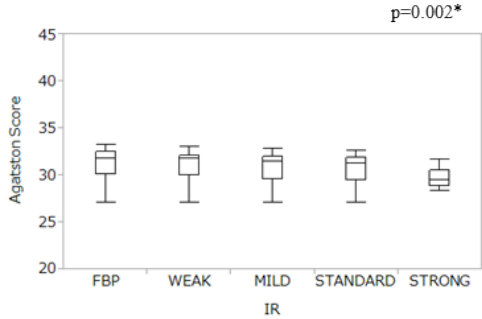

d) $70 \mathrm{~mA}$ ( $71 \%$ dose reduction)

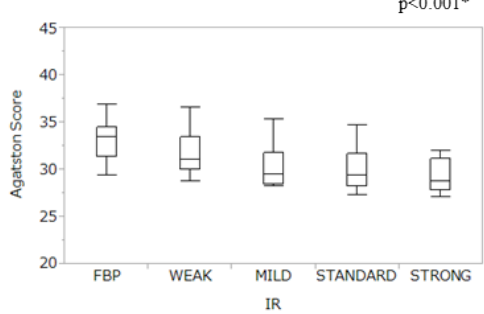

b) $150 \mathrm{~mA}(38 \%$ dose reduction)

$\mathrm{p}<0.001^{*}$

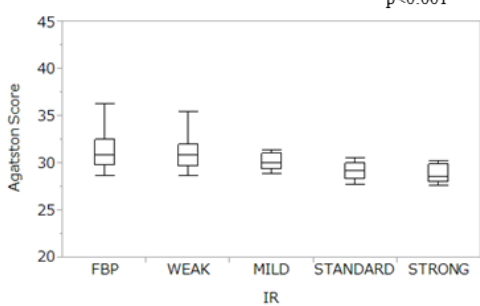

e) $60 \mathrm{~mA}(75 \%$ dose reduction)

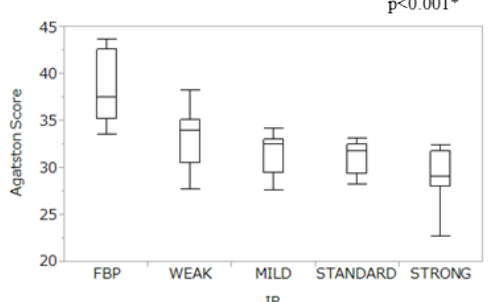

c) $100 \mathrm{~mA}(58 \%$ dose reduction)

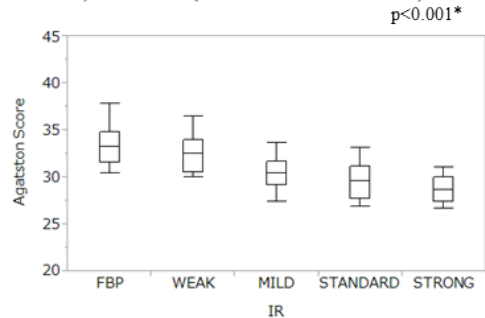

IR

* Jonckheere-Terpstra test

Figure 3. Agatston scores for various IR levels when radiation dose was held constant 
significantly decreased as IR levels increased ( $\mathrm{p}<0.003$, respectively).

Agatston scores for various radiation dose and IR levels, compared to control (FBP at 240mA) (Table

\section{1, Figure 4)}

When the FBP protocol was used, Agatston scores significantly increased for some phantom sizes at $58 \%, 71 \%$, and $75 \%$ reductions in radiation dose relative to scores obtained when routine doses were utilized in the FBP protocol.

When the AIDR3D: Weak protocol was used, Agatston scores significantly increased for some phantom sizes at $71 \%$ or $75 \%$ reductions in radiation dose relative to scores obtained when routine doses were utilized in the FBP protocol. When the AIDR3D: Mild protocol was used, no significant differences in Agatston scores were observed between IR and FBP protocols for any phantom size.

When the AIDR3D: Standard protocol was used, significantly higher average Agatston scores were observed in some phantom sizes at a $38 \%$ reduction in radiation dose, although no significant differences were observed for any phantom size when other dose levels were utilized. When the AIDR3D: Strong protocol was used, Agatston scores significantly decreased for some phantom sizes relative to scores obtained when routine doses were utilized in the FBP protocol.

\section{Discussion}

We can reduce the radiation dose of coronary CT angiography by using AIDR3D, and recent studies have reported that the mean effective radiation dose was $2.55+/-1.21 \mathrm{mSv}$, using AIDR3D. ${ }^{15)}$ On the other hand, the usual radiation dose of CAC scoring without iterative reconstruction is $1-2 \mathrm{mSv}$. However, we have had difficulties using AIDR3D in CAC scanning because the effects of AIDR3D remained unclear.

To the best of our knowledge, the present study is the first to evaluate the effects of dose reduction and iterative reconstruction on CAC score using a pulsating cardiac phantom. Our results indicate that Agatston scores for each calcium phantom tended to increase when lower doses of radiation $(58 \%, 71 \%$ and $75 \%$ reduced) were used, while they tended to decrease when higher IR levels were used, when compared with FBP. Furthermore, no significant differences in Agatston scores were observed between routine-dose FBP protocol and 75\%-reduced dose AIDR3D: Mild and AIDR3D: Standard protocols.

Though research indicates that decreased exposure to radiation occurs when the ALARA principle
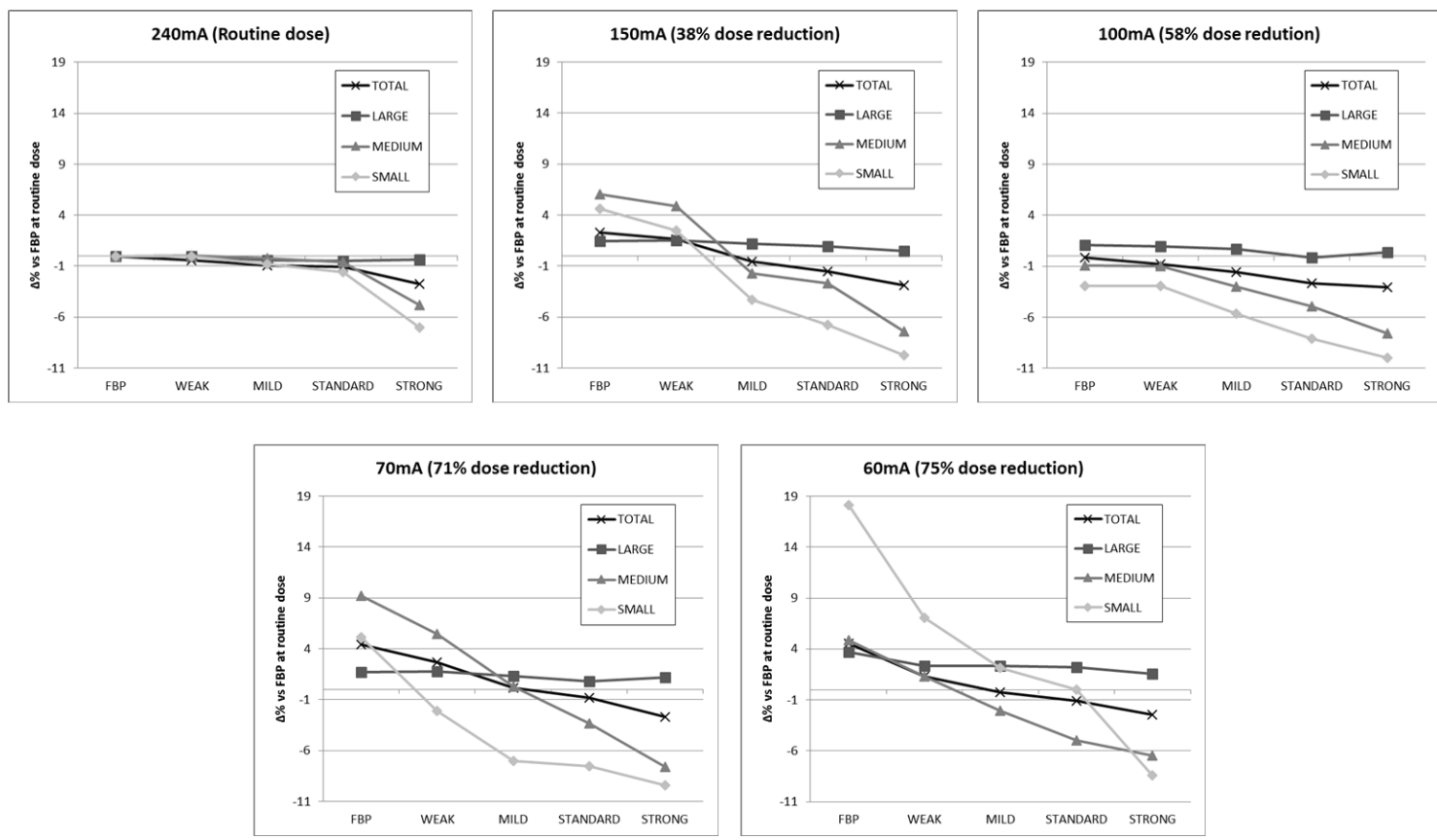

Figure 4. Median percentage difference in Agatston score between reconstruction methods at each radiation dose (tube current) relative to the routine dose of the FBP protocol. 
is followed, reducing the radiation dose when a standard FBP protocol is utilized may lead to increased image noise ${ }^{16)}$ and overestimation of the Agatston score. ${ }^{17)}$ However, IR algorithms introduced from some vendors ${ }^{17)}$ represent new techniques designed to reduce image noise and improve image quality using low doses of radiation. ${ }^{8-10)}$ An in vivo study by Kurata et al. revealed that Agatston scores tend to decrease as sonogram affirmed iterative reconstruction (SAFIRE) levels increase. ${ }^{18)}$ Our observation that Agatston scores tend to decrease as IR levels increase further support these conclusions. The results of the present study, therefore, suggest that similar Agatston scores may be obtained when either standard-dose FBP or low-dose IR protocols are utilized.

Previous research supports the use of certain combinations of dose reduction and IR levels. Murazaki et al. investigated the effect of hybrid iterative reconstruction (iDose) on Agatston scores in an in vitro study using a static cardiac phantom and concluded that radiation dose can be reduced up to $50 \%$ using the iDose technique. ${ }^{19)}$ In vitro studies by Blobel et al. ${ }^{11)}$ and Tatsugami et al. ${ }^{20)}$ revealed that the use of AIDR3D allowed for respective reductions in radiation doses of up to $80 \%$ and $67 \%$ without impairing the quantification of Agatston scores, relative to doses utilized in routine protocols, using the static anthropomorphic phantom. Other researchers have concluded that IR allows for up to an $82 \%$ reduction in radiation dose during coronary calcium scoring in ex vivo heart studies when compared with standarddose FBP. ${ }^{1213)}$ In the present study, we investigated the effects of various combinations of dose reduction and IR levels on Agatston scores using a pulsating cardiac phantom to simulate motion in the beating heart. In accordance with the results of the aforementioned studies, our findings indicate that AIDR3D protocols allow for up to $75 \%$ reductions in radiation dose when mild or standard IR levels are utilized, under the effect of cardiac motion.

The present study possesses some limitations. First, as the pulsating phantom system was set to 60 bpm, we did not assess the effect of AIDR3D on motion artifacts under different heart rate conditions. Second, we evaluated only Agatston scores, and therefore, the effect of AIDR3D on other calcium scores, such as mass score and volume score, were still uncertain. Third, as our investigation involved $e x$ vivo analysis of low to relatively high Agatston scores, we did not assess the effects on very small
Agatston scores/non-calcified plaques. Furthermore, the calcium phantoms utilized in the present study may have been larger than real arterial calcium deposits, thus altering the influence of AIDR3D. Therefore, future research should utilize much smaller calcium phantoms in order to clarify this point. In addition, the applicability of our study to different vendors of CT scanners may be limited, as each CT vendor has developed a unique IR technique. Nonetheless, the results of the present study indicate that IR techniques may allow for reductions in radiation dose when evaluating Agatston scores.

\section{Conclusion}

In the present study, we observed that mild and standard AIDR3D protocols allowed for significant reductions in radiation dose relative to standard FBP protocols, without influencing Agatston scores. Therefore, evaluation of CAC score using a combination of low-dose radiation and AIDR3D may present a reliable alternative to conventional CAC scoring.

\section{References}

1) World Health Organization Cardiovascular diseases. Fact sheet no. 317. http://www.who.int/ mediacentre/factsheets/fs317/en/index.html.

2) Agatston AS, Janowitz WR, Hildner FJ, Zusmer NR, Viamonte M Jr, Detrano R. Quantification of coronary artery calcium using ultrafast computed tomography. J Am Coll Cardiol 1990; 15: 827-832.

3) Greenland P, Bonow RO, Brundage BH, Budoff MJ, Eisenberg MJ, Grundy SM, Lauer MS, Post WS, Raggi P, Redberg RF, Rodgers GP, Shaw LJ, Taylor AJ, Weintraub WS. ACCF/AHA 2007 clinical expert consensus document on coronary artery calcium scoring by computed tomography in global cardiovascular risk assessment and in evaluation of patients with chest pain: a report of the American College of Cardiology Foundation Clinical Expert Consensus Task Force (ACCF/AHA Writing Committee to Update the 2000 Expert Consensus Document on Electron Beam Computed Tomography) developed in collaboration with the Society of Atherosclerosis Imaging and Prevention and the Society of Cardiovascular Computed Tomography. J Am Coll Cardiol 2007; 49: 378-402.

4) Greenland P, LaBree L, Azen SP, Doherty TM, Detrano RC. Coronary artery calcium score combined with Framingham score for risk pre- 
diction in asymptomatic individuals. JAMA. 2004; 291: 210-215.

5) Kim KP, Einstein AJ, Berrington de Gonzalez A. Coronary artery calcification screening: estimated radiation dose and cancer risk. Arch Intern Med 2009; 169: 1188-1194.

6) Einstein AJ, Moser KW, Thompson RC, Cerqueira MD, Henzlova MJ. Radiation dose to patients from cardiac diagnostic imaging. Circulation. 2007; 116: 1290-1305.

7) Greenland P, Alpert JS, Beller GA, Benjamin EJ, Budoff MJ, Fayad ZA, Foster E, Hlatky MA, Hodgson JM, Kushner FG, Lauer MS, Shaw LJ, Smith SC Jr, Taylor AJ, Weintraub WS, Wenger NK, Jacobs AK, Smith SC Jr, Anderson JL, Albert N, Buller CE, Creager MA, Ettinger SM, Guyton RA, Halperin JL, Hochman JS, Kushner FG, Nishimura R, Ohman EM, Page RL, Stevenson WG, Tarkington LG, Yancy CW. 2010 ACCF/AHA guideline for assessment of cardiovascular risk in asymptomatic adults: a report of the American College of Cardiology Foundation/ American Heart Association Task Force on Practice Guidelines. J Am Coll Cardiol 2010; 56: e50-e103.

8) Gervaise A, Osemont B, Lecocq S, Noel A, Micard E, Felblinger J, Blum A. CT image quality improvement using adaptive iterative dose reduction with wide volume acquisition on 320detector CT. Eur Radiol 2012; 22: 295-301.

9) Hara AK, Paden RG, Silva AC, Kujak JL, Lawder HJ, Pavlicek W. Iterative reconstruction technique for reducing body radiation dose at CT: feasibility study. AJR Am J Roentgenol 2010; 193: 764-771.

10) Liu YJ, Zhu PP, Chen B, Wang JY, Yuan QX, Huang WX, Shu H, Li ER, Liu XS, Zhang K, Ming $\mathrm{H}, \mathrm{Wu} \mathrm{ZY}$. A new iterative algorithm to reconstruct the refractive index. Phys Med Biol 2007; 52: L5-L13.

11) Blobel J, Mews J, Schuijf JD, Overlaet W. Determining the radiation dose reduction potential for coronary calcium scanning with computed tomography: an anthropomorphic phantom study comparing filtered backprojection and the adaptive iterative dose reduction algorithm for image reconstruction. Investigate Radiology 2013; 48: 857-862.

12) den Harder AM, Willemink MJ, Bleys RL, de Jong PA, Budde RP, Schilham AM, Leiner T. Dose reduction for coronary calcium scoring with hybrid and model-based iterative reconstruction: an ex vivo study. Int $\mathrm{J}$ Cardiovasc Imaging 2014: 30: 1125-1133.

13) Willemink MJ, Takx RA, de Jong PA, Budde RP, Bleys RL, Das M, Wildberger JE, Prokop M, Buls N, de Mey J, Schilham AM, Leiner T. The impact of CT radiation dose reduction and iterative reconstruction algorithms from four different vendors on coronary calcium scoring. European Radiology 2014; 24: 2201-2212.

14) Arbab-Zadeh A, Texter J, Ostbye KM, Kitagawa K, Brinker J, George RT, Miller JM, Trost JC, Lange RA, Lima JA, Lardo AC. Quantification of lumen stenoses with known dimensions by conventional angiography and computed tomography: implications of using conventional angiography as gold standard. Heart 2010; 96: 1358-1363.

15) Szilveszter B, Elzomor H, Károlyi M, Kolossváry $M$, Raaijmakers $R$, Benke $K$, Celeng $C$, Bartykowszki A, Bagyura Z, Lux Á, Merkely B, Maurovich-Horvat P. The effect of iterative model reconstruction on coronary artery calcium quantification. Int J Cardiovasc Imaging. 2016; 32: 153-160.

16) Marwan M, Mettin C, Pflederer T, Seltmann M, Schuhbäck A, Muschiol G, Ropers D, Daniel WG, Achenbach S. Very low-dose coronary artery calcium scanning with high-pitch spiral acquisition mode: comparison between $120-\mathrm{kV}$ and $100-\mathrm{kV}$ tube voltage protocols. J Cardiovasc Comput Tomogr 2013; 7: 32-38.

17) Willemink MJ, de Jong PA, Leiner T, de Heer LM, Nievelstein RA, Budde RP, Schilham AM. Iterative reconstruction techniques for computed tomography Part 1: technical principles. Eur Radiol 2013; 23: 1623-1631.

18) Kurata A, Dharampal A, Dedic A, de Feyter PJ, Krestin GP, Dijkshoorn ML, Nieman K. Impact of iterative reconstruction on CT coronary calcium quantification. Eur Radiol 2013; 23: 32463252.

19) Murazaki H, Funama Y, Hatemura M, Fujioka C, Tomiguchi S. Quantitative evaluation of calcium (content) in the coronary artery using hybrid iterative reconstruction (iDose)algorithm on low-dose 64-detector CT: comparison of iDose and filtered back projection. Nihon Hoshasen Gijutsu Gakkai Zasshi 2011; 67: 360-366.

20) Tatsugami F, Higaki T, Fukumoto $W$, Kaichi $Y$, Fujioka C, Kiguchi M, Yamamoto H, Kihara Y, 
Awai K. Radiation dose reduction for coronary artery calcium scoring at 320-detector CT with adaptive iterative dose reduction 3D. Int J Cardiovasc Imaging. 2015; 31: 1045-1052. 